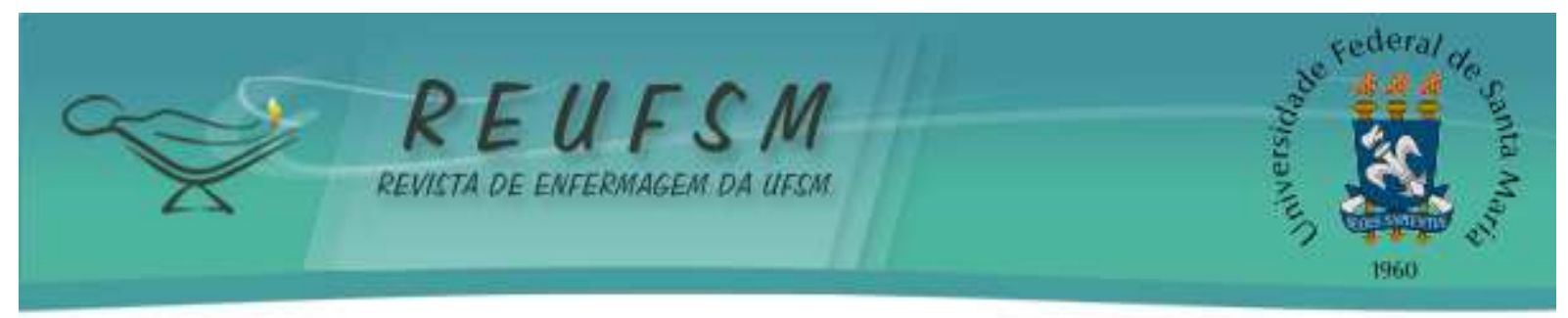

ARTIGO ORIGINAL

\title{
HIPODERMÓCLISE EM PACIENTES COM CÂNCER EM CUIDADOS PALIATIVOS
}

\section{HYPODERMOCLYSIS IN CANCER PATIENTS IN PALLIATIVE CARE HIPODERMOCLISIS EN PACIENTES CON CÁNCER EN CUIDADOS PALIATIVOS}

\author{
Gislene Pontalti ${ }^{1}$ \\ Caren de Oliveira Riboldi ${ }^{2}$ \\ Luciana dos Santos ${ }^{3}$ \\ Vanessa Kenne Longaray ${ }^{4}$ \\ Desirée Amorim Guzzo 5 \\ Isabel Cristina Echer ${ }^{6}$
}

Doi: $10.5902 / 2179769228551$

RESUMO: Objetivo: analisar o uso da hipodermóclise em pacientes com câncer em cuidados paliativos. Método: estudo transversal descritivo, com objetivo de descrever a experiência da utilização da hipodermóclise em pacientes sob cuidados paliativos, realizado em um hospital universitário do sul do Brasil. A amostra, por conveniência, abrangeu 80 pacientes que internaram em cuidados paliativos entre março/2014 e março/2015. A coleta de dados ocorreu mediante instrumento específico e a análise por estatística descritiva. Resultados: as neoplasias predominantes foram pâncreas $9(11,3 \%)$, intestino $8(10 \%)$, pulmão $8(10 \%)$ e gástrica $8(10 \%)$. Entre as indicações para hipodermóclise prevaleceram analgesia 63(78,8\%), rede venosa precária $51(63,8 \%)$ e intolerância oral 38(47,5\%). Dos 21 fármacos prescritos e administrados destacam-se morfina 76(95,0\%), metoclopramida 49(61,3\%), dipirona 39(48,8\%), ondansetrona $29(36,3 \%)$ e dexametasona 12(15,0\%). Ocorreram 105 punções e nenhuma complicação sistêmica. Considerações finais: a hipodermóclise mostrou-se uma terapêutica medicamentosa eficaz, segura e menos invasiva na prática clínica paliativista.

Descritores: Hipodermóclise; Cuidados Paliativos; Administração de Terapia Medicamentosa

ABSTRACT: Aim: to analyze the use of hypodermoclysis in cancer patients in palliative care. Method: descriptive, cross - sectional study, with the purpose of describing the experience in patients undergoing palliative care, performed at a university hospital in southern Brazil. The sample consisted of 80 patients hospitalized in palliative care between March 2014 and March 2015. The data collection was made by specific instrument and the analysis was made through descriptive statistics. Results: pancreas $9(11.3 \%)$, intestine $8(10 \%)$, lung $8(10 \%)$ and gastric $8(10 \%)$ were the predominant neoplasms. Among the indications for hypodermoclysis, there

\footnotetext{
${ }^{1}$ Enfermeira. Mestre em Gerência em Serviço. Hospital de Clínicas de Porto Alegre (HCPA). Porto Alegre, RS, Brasil. E-mail: gislene818@gmail.com

${ }^{2}$ Enfermeira. Mestre em Enfermagem. HCPA. Porto Alegre, RS, Brasil. E-mail: criboldi@ hcpa.edu.br

${ }^{3}$ Farmacêutica. Mestre em Ciências Farmacêuticas. HCPA. Porto Alegre, RS, Brasil. Email: lusantos@hcpa.edu.br

${ }^{4}$ Enfermeira. Mestre em Psiquiatria. HCPA. Porto Alegre, RS, Brasil. E-mail: vlongaray@ hcpa.edu.br

${ }^{5}$ Enfermeira. Graduada em Enfermagem pela Universidade Federal do Rio Grande do Sul (UFRGS). Porto Alegre, RS, Brasil. E-mail: desiree.amorim@hotmail.com

${ }^{6}$ Enfermeira. Doutora em Ciências Médicas, Professora Associada da Escola de Enfermagem, UFRGS. Porto Alegre, RS, Brasil. E-mail: isabelecher@gmail.com
} 


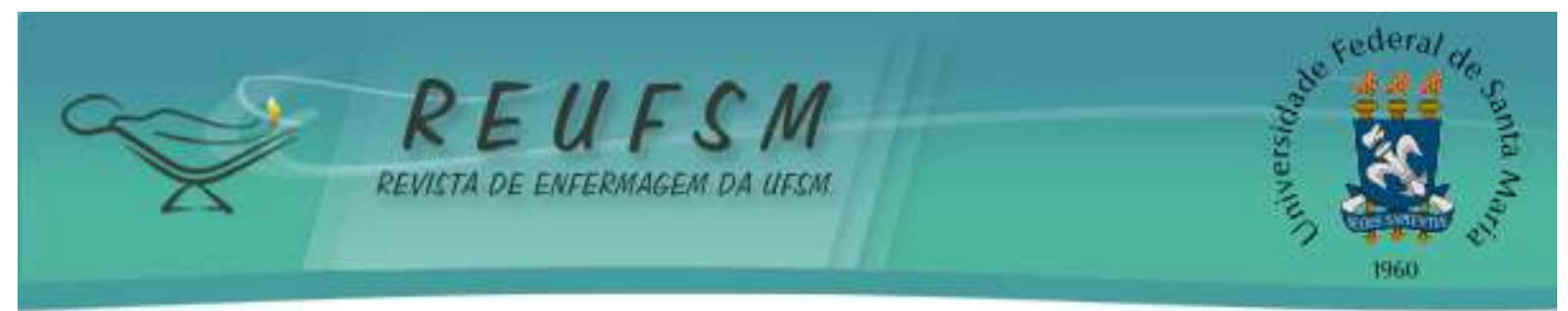

were analgesia 63 (78.8\%), precarious venous network 51 (63.8\%) and oral intolerance 38 (47.5\%). Of the 21 drugs prescribed and administered, the following stand out: morphine 76 (95.0\%), metoclopramide 49 (61.3\%), dipyrone 39 (48.8\%), ondansetron 29 (36.3\%) and dexamethasone 12 (15,0\%). There were 105 punctures and no systemic complications. Final considerations: hypodermoclysis has been shown to be an effective, safe and less invasive drug combination in palliative clinical practice.

Descriptors: Hypodermoclisis; Palliative care; Administration of medicinal therapy

RESUMEN: Objetivo: analizar el uso de la hipodermoclisis en pacientes con cáncer en cuidados paliativos. Método: estudio transversal descriptivo, en pacientes que utilizan hipodermoclisis en cuidados paliativos, realizado en un hospital universitario del sur de Brasil. La muestra, por conveniencia, comprendió 80 pacientes que ingresaron en cuidados paliativos entre marzo de 2014 y marzo de 2015. La recolección de datos ocurrió pode medio de un instrumento específico y el análisis por estadística descriptiva. Resultados: las neoplasias predominantes fueron páncreas 9 (11,3\%), intestino $8(10 \%)$, pulmón $8(10 \%)$ y gástrica 8 (10\%). Entre las indicaciones para hipodermoclisis prevalecieron analgesia 63 (78,8\%), red venosa precaria 51 (63,8\%) e intolerancia oral 38 (47,5\%). De los 21 fármacos prescritos y administrados, se pueden destacar morfina 76(95,0\%), metoclopramina 49(61,3\%), dipirona 39(48,8\%), ondansetrona 29(36,3\%) y dexametasona 12(15,9\%). En el período ocurrieron 105 punciones y ninguna complicación sistémica. Consideraciones finales: la hipodermoclisis se mostró una terapia medicamentosa eficaz, segura y menos invasiva en la práctica clínica de los cuidados paliativos.

Descriptores: Hipodermóclisis; Cuidados paliativos; Administración de terapia medicamentosa

\section{INTRODUÇÃO}

Hipodermóclise ou terapia subcutânea é uma técnica centenária que consiste na infusão de fluidos e fármacos para o espaço subcutâneo, em situações não emergenciais. Até a década de 1950 foi amplamente utilizada, porém em virtude do desconhecimento de fármacos que resultavam em reações adversas severas, da utilização de solutos hipotônicos que provocavam choque hipovolêmico e com a introdução da canalização endovenosa pela medicina dos Estados Unidos, esta terapêutica passou a ser pouco empregada. ${ }^{1-5}$

Nos anos 1960, no Reino Unido, o desenvolvimento dos cuidados paliativos fez com que a hipodermóclise ressurgisse enquanto técnica para os desafios da paliação no controle de sintomas em pacientes adultos e pediátricos. ${ }^{5-7}$ As situações em que comumente utiliza-se a via subcutânea relacionam-se ao comprometimento da via oral por disfagia, vômitos incoercíveis e obstrução gastrintestinal, prejuízo cognitivo por agitação e desorientação, rede endovenosa inacessível por caquexia, desidratação e agentes esclerosantes. ${ }^{1,7-9}$ 


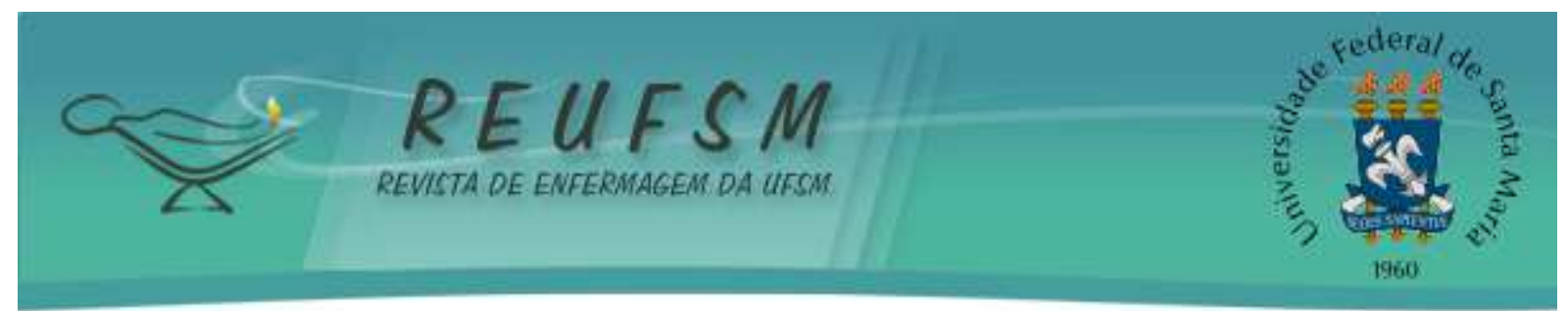

No Brasil, os serviços de cuidados paliativos têm seu desenvolvimento desde o final da década de 1990, quando o Instituto Nacional de Câncer (INCA) do Ministério da Saúde, inaugurou em 1998 o hospital Unidade IV, exclusivamente dedicado aos Cuidados Paliativos. Desde então, esses locais se encontram em processo dinâmico e franca expansão derivados, principalmente, de serviços de controle da dor e cuidados paliativos em hospitais gerais e em programas de atendimento domiciliar. ${ }^{2,9-10}$

$\mathrm{O}$ aumento da expectativa de vida, as mudanças no perfil populacional quanto à cronicidade das doenças, o reconhecimento das instituições governamentais e os esforços de gestores e profissionais da saúde para implantar serviços com leitos para internação, consultas ambulatoriais e atendimento domiciliar destinado aos cuidados paliativos no setor público e privado, têm reiterado a importância e necessidade deste tipo de cuidado para a sociedade. ${ }^{10-13}$

O Instituto Nacional de Câncer emprega a hipodermóclise desde 1999 e em 2009 publicou o manual de terapia subcutânea, validando a terapêutica como um método acessível e bem tolerado pelos pacientes, com poucos efeitos adversos, de baixo custo e fácil manipulação, o que favorece a continuidade no ambiente domiciliar por um cuidador capacitado pela equipe multiprofissional. ${ }^{2-3,6-7,11-12}$

Estudos recentes ${ }^{2,4,8,12,14}$ têm relatado que a hipodermóclise está em crescente utilização como via parenteral de eleição em pacientes sob cuidados paliativos, embora sua aplicação ainda seja variável nos diversos centros brasileiros. Os relatos científicos, ensaios clínicos randomizados e controlados sobre a terapêutica ainda são incipientes, persistindo dúvidas quanto a utilização no que tange seleção de fármacos administrados, tempo de permanência do dispositivo e complicações inerentes ao procedimento. Assim, este estudo busca responder a seguinte questão de pesquisa: a hipodermóclise consiste em uma terapêutica medicamentosa eficaz, segura e menos invasiva na prática clínica em cuidados paliativos?

Neste contexto, o presente estudo objetivou analisar o uso da hipodermóclise em pacientes com câncer em cuidados paliativos, buscando estabelecer diretrizes para o cuidado com foco na qualidade e segurança da técnica na prática paliativista.

\section{MÉTODO}

Estudo transversal descritivo, realizado em uma unidade de cuidados paliativos de um hospital universitário do sul do Brasil, a qual dispõe de sete leitos destinados a pacientes 


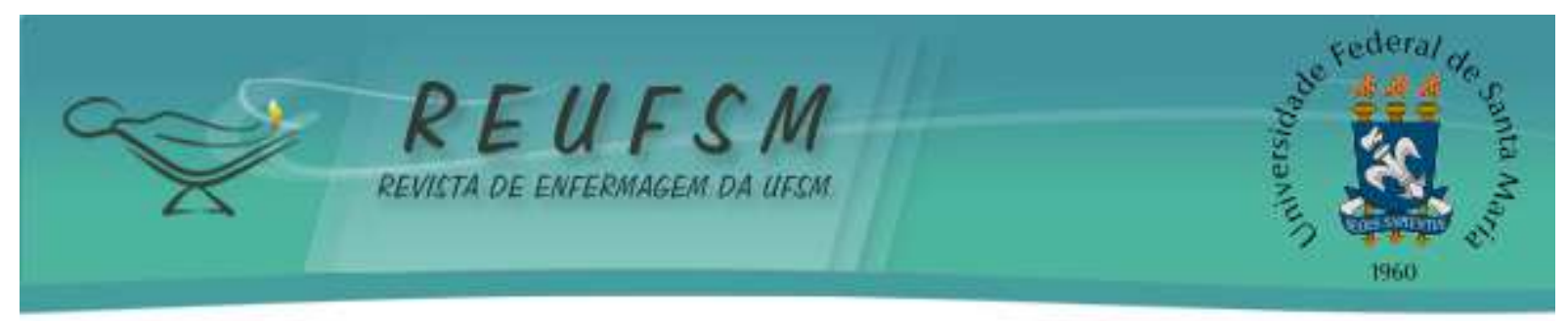

adultos com câncer, provenientes do Sistema Único de Saúde. Os critérios para internação nesta unidade foram definidos no Plano Assistencial aos Pacientes em Cuidados Paliativos, da instituição, e compreende não apresentar alteração no nível de consciência (coma ou torpor), indicação de cuidados paliativos com registro em prontuário, concordância da família e/ou paciente sobre a internação e disponibilidade de acompanhante 24 horas.

A população do estudo foi composta pelos pacientes que internaram no período de março de 2014 a março de 2015. A amostra selecionada por conveniência, abrangeu 80 pacientes com prescrição médica de infusão de medicamentos por via subcutânea. Como critério de inclusão adotou-se os pacientes puncionados com dispositivo de material flexível de Vialon, calibre 22G, preconizado pela instituição que foi campo de estudo. Pacientes que foram puncionados com outro tipo de dispositivo foram excluídos do estudo.

$\mathrm{Na}$ instituição, a hipodermóclise é um procedimento realizado pelo enfermeiro, cuja técnica consiste em inserir uma agulha hipodérmica no tecido subcutâneo. $\mathrm{O}$ dispositivo apresenta formato de borboleta que facilita a inserção da agulha de forma menos traumática, favorecendo a fixação na pele do paciente e reduzindo o risco de lesão quando comparado com outro artefato no tecido subcutâneo. ${ }^{15}$

Os locais utilizados para hipodermóclise foram os indicados pela literatura como região anterior do tórax e escapular, quadrantes abdominais, laterais das coxas e região do deltóide. A permanência do dispositivo é de, aproximadamente, 96 horas, podendo ser maior se não apresentar sinais de infecção. Para amenizar o dano ao tecido, foi realizado rodízio dos locais de punção, mantendo uma distância de cinco centímetros do último sítio puncionado conforme determina a literatura. . $^{3,7-8,11-12}$

A coleta de dados foi realizada diariamente com o preenchimento de um instrumento pelos enfermeiros previamente capacitados, que contemplava 11 questões objetivas e de escolha múltipla, relacionadas à caracterização do paciente, indicação para hipodermóclise, medicações utilizadas, sítio de punção, tempo de permanência do cateter, reações adversas e motivo de retirada. Esse instrumento foi construído pelos pesquisadores e testado previamente por meio de um piloto para avaliar a sua adequabilidade.

Os dados coletados foram organizados em planilha do Microsoft Excel for Windows ${ }^{\circledR} \mathrm{e}$ analisados com auxílio do Statistical Package for Social Sciences (SPSS) versão 18.0, por meio de estatística descritiva. 


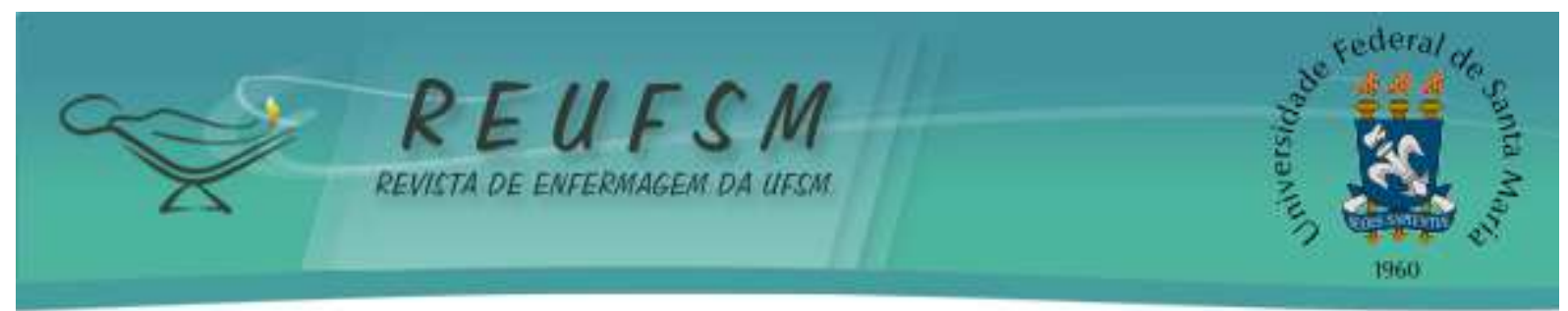

O estudo foi aprovado pelo Comitê de Ética em Pesquisa da instituição, campo de estudo, sob o número de protocolo 13-0358, em janeiro de 2014. Todos os participantes preencheram Termo de Consentimento Livre e Esclarecido de acordo com a Resolução 466/2012 do Conselho Nacional de Saúde. ${ }^{16}$

\section{RESULTADOS}

Participaram do estudo 80 pacientes com distribuição uniforme para ambos os sexos. A caracterização dos pacientes e o tipo de neoplasia primária estão descritos na Tabela 1.

Tabela 1 - Características dos pacientes quanto ao sexo, idade e tipo de neoplasia primária.

\begin{tabular}{lll}
\hline Variáveis & $\mathrm{N}=80$ & $(\%)$ \\
\hline Sexo & & \\
Feminino & 40 & 50 \\
Idade* & $62,3 \pm 13,78$ & \\
Tipo de neoplasia primária** & & \\
Pâncreas & 9 & 11,3 \\
Gástrica & 8 & 10,0 \\
Intestinal & 8 & 10,0 \\
Pulmão & 8 & 10,0 \\
Cabeça e pescoço & 7 & 8,8 \\
Mama & 7 & 8,8 \\
Esôfago & 6 & 7,5 \\
Ovário & 4 & 5,0 \\
Renal & 4 & 5,0 \\
Melanoma & 3 & 3,8 \\
Sistema nervoso central & 3 & 3,8 \\
Colo uterino & 2 & 2,5 \\
Hepática & 2 & 2,5 \\
Óssea & 2 & 2,5 \\
Outros & 6 & 7,5 \\
\hline
\end{tabular}

*Variável contínua expressa em média e desvio padrão

**Escolha múltipla

Na Tabela 2 visualizam-se os motivos de internação hospitalar dos pacientes na unidade de cuidados paliativos e as indicações para a terapia hipodermóclise.

Tabela 2 - Motivo de internação dos pacientes na unidade de cuidados paliativos e indicação para hipodermóclise.

\begin{tabular}{lcc}
\hline Variáveis & $\mathrm{N}=80$ & $(\%)$ \\
\hline Motivo de internação hospitalar* & & \\
Dor não controlada & 47 & 58,8 \\
Náuseas e vômitos & 30 & 37,5 \\
Dispneia & 24 & 30,0 \\
Desorientação & 22 & 27,5 \\
Fadiga & 16 & 20,0 \\
Inapetência & 15 & 18,8 \\
\hline
\end{tabular}




\begin{tabular}{|c|c|c|}
\hline$R_{\text {REVISTA D }}$ & $\frac{8}{5}$ & $\frac{c_{e}}{3}$ \\
\hline Anorexia & 11 & 13,8 \\
\hline Agitação & 7 & 8,8 \\
\hline Constipação & 6 & 7,5 \\
\hline Obstrução intestinal & 6 & 7,5 \\
\hline Febre & 3 & 3,8 \\
\hline Sangramento & 2 & 3,8 \\
\hline Outros & 7 & 8,8 \\
\hline \multicolumn{3}{|l|}{ Indicação para hipodermóclise* } \\
\hline Analgesia fixa & 63 & 63,8 \\
\hline Rede venosa inacessível & 51 & 47,5 \\
\hline Intolerância da via oral & 38 & 11,3 \\
\hline Alteração do nível de consciência & 9 & 6,3 \\
\hline Sedação final & 5 & 1,3 \\
\hline Solicitação do paciente & 1 & 1,3 \\
\hline Alta domiciliar & 1 & 1,3 \\
\hline
\end{tabular}

*Escolha múltipla

Foram prescritos e administrados por hipodermóclise 21 fármacos diferentes, de forma intermitente ou contínua, por infusão ou em bolus, conforme prescrição médica e recomendações da tabela de medicamentos institucional para uso desta via. $\mathrm{Na}$ Tabela 3 constam as medicações prescritas e administradas nos pacientes, sendo que a terapia medicamentosa abrangeu mais de um fármaco concomitante durante a internação.

Tabela 3 - Fármacos prescritos e administrados por hipodermóclise nos pacientes.

\begin{tabular}{lcc}
\hline Variáveis & $\mathrm{N}=80$ & $(\%)$ \\
\hline Medicações prescritas e administradas por hipodermóclise* & \\
Morfina & 76 & 95,0 \\
Metoclopramida & 49 & 61,3 \\
Dipirona & 39 & 48,8 \\
Ondansetrona & 29 & 36,3 \\
Dexametasona & 12 & 15,0 \\
Ranitidina & 11 & 13,8 \\
Haloperidol & 9 & 11,3 \\
Solução glicofisiológica & 10,0 \\
Solução fisiológica 0,9\% & 8 & 10,0 \\
Eletrólitos (cloreto de potássio 10\% e sódio 20\%) & 8 \\
Midazolam & 5 & 6,3 \\
Cetamina & 5,0 \\
Escopolamina & 4 & 5,0 \\
Furosemida & 3,8 \\
Cefepima & 3 & 3,8 \\
Clorpromazina & 3 & 3,8 \\
Dimenidrinato & 2,8 \\
Octreotida & 2,5 \\
Solução glicosada 5\% & 2,5 \\
Omeprazol & 2 & 2,5 \\
Ampicilina & 1,3 \\
\hline Escolha múlipla & 1,3 \\
\hline
\end{tabular}




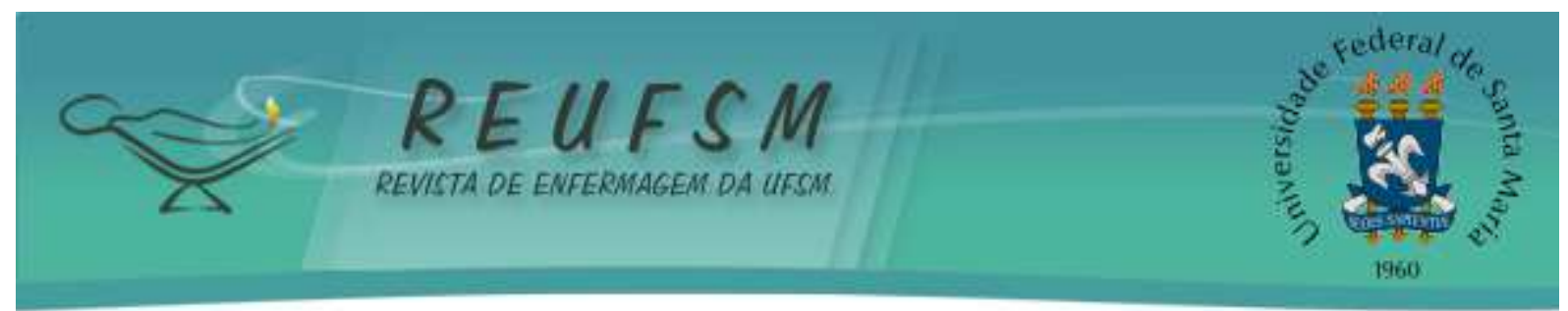

O sítio de punção e tempo de permanência do cateter para hipodermóclise, complicações locais e motivos de retirada do dispositivo estão apresentados na tabela 4 .

Tabela 4 - Sítio de punção e tempo de permanência do cateter para hipodermóclise, complicações locais e motivo de retirada do dispositivo.

\begin{tabular}{lll}
\hline Variáveis & $\mathrm{N}=105$ & $(\%)$ \\
\hline Sítio de punção do cateter para hipodermóclise* & & \\
Tórax anterior esquerdo & 55 & 52,4 \\
Tórax anterior direito & 37 & 35,2 \\
Abdômen & 4 & 3,8 \\
Parte anterior e superior braço esquerdo & 3 & 2,9 \\
Parte anterior e superior braço direito & 2 & 1,9 \\
Parte anterior coxa esquerda & 2 & 1,9 \\
Parte anterior coxa direita & 2 & 1,9 \\
Tempo de permanência do cateter para hipodermóclise & & \\
Média em dias & $7,25 \pm 5,10$ & \\
Complicações locais & 4 & 3,8 \\
Edema & 4 & 3,8 \\
Extravazamento de líquidos & 4 & 3,8 \\
Dor & 4 & 3,8 \\
Rubor e hiperemia & 1 & 1,0 \\
Intumescimento & 1 & 1,0 \\
Sangramento discreto & & \\
Motivo de retirada do dispositivo & 62 & 59,0 \\
Óbito & 18 & 17,2 \\
Complicações locais & 10 & 9,5 \\
Suspensão do tratamento medicamentoso & 9 & 8,6 \\
Alta hospitalar & 6 & 5,7 \\
Saída acidental & \\
\hline
\end{tabular}

*Escolha múltipla

\section{DISCUSSÃO}

No Brasil, estima-se que entre 2014 e 2015 tenham surgido, aproximadamente, 576 mil casos novos de câncer. Os tipos de câncer mais incidentes na região sul entre os homens são próstata, pulmão, cólon e reto, estômago e esôfago. Entre as mulheres destacam-se mama, cólon e reto, pulmão, tireóide e útero. ${ }^{17}$ Neste estudo, a amostra apresentou distribuição uniforme em relação ao sexo e constatou-se a prevalência de neoplasias gástrica de intestino, pulmão, mama e esôfago.

A idade avançada dos pacientes da amostra reforça as estimativas demográficas que apontam para o aumento da expectativa de vida e o envelhecimento populacional, colocando as doenças crônico-degenerativas como uma das prioridades de atenção na área da saúde. 9,12,14,18 


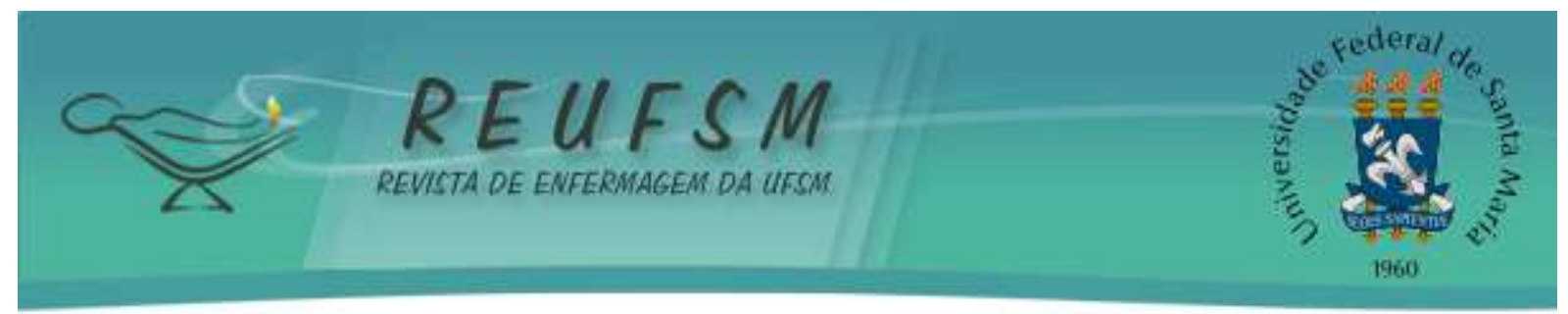

Pacientes em cuidados paliativos comumente necessitam de hospitalização para o controle da dor, náuseas e vômitos, dispneia e outros sintomas provenientes da doença e que interferem no conforto e qualidade de vida, conforme se observou no presente estudo. A via oral, embora seja a opção inicial para tratamento, nem sempre está disponível pelas condições clínicas do paciente, o que justifica a escolha da hipodermóclise, ${ }^{3,8,11,14,18-19}$ corroborando com os achados.

No presente estudo, as medicações mais utilizadas pela via subcutânea foram morfina e metoclopramida, respectivamente, o que vem ao encontro dos principais sintomas apresentados pelos pacientes participantes da pesquisa. Estudos ${ }^{8,20-22}$ apontam que a morfina, um analgésico opióide, é o fármaco de escolha para o controle da dor e dispneia, apresentando ação benéfica no alívio destes sintomas em cuidados paliativos.

As soluções fisiológicas, glicofisiológicas e com eletrólitos, utilizadas para hidratação do paciente, foram administradas por infusão contínua. Da mesma maneira, a morfina e a cetamina, quando a finalidade foi sedoanalgesia. Os demais fármacos foram administrados em bolus, diluídos conforme a tabela de medicamentos validada na instituição. ${ }^{1,8-9,11,13,16}$ Esses resultados evidenciam os benefícios da terapia por hipodermóclise, uma vez que os pacientes podem preservar a terapia medicamentosa não sendo submetidos a múltiplas punções venosas e/ou procedimentos invasivos, como a colocação de acesso venoso central.

No Brasil, a maioria dos fármacos por hipodermóclise é de uso off-label, ou seja, sua indicação não consta em bulas ou protocolos, sendo a administração baseada nas práticas assistenciais das instituições, a critério do prescritor. Alguns destes fármacos apresentam contraindicações por apresentarem risco de causar irritação ou necrose tecidual, justificado pelo pH extremo (muito ácido ou muito alcalino), osmolaridade e características de solubilidade da formulação ou solução. ${ }^{1,11,19,20-23}$

No que se refere à administração do dimenidrinato por hipodermóclise, na literatura não se observam evidências seguras para seu uso, sendo substituído por metoclopramida ou ondansetrona. A sua utilização, entretanto, não está contraindicada, podendo ser administrado com precaução desde que não seja misturado em seringa com outro fármaco. ${ }^{23}$ No presente estudo identificou-se que esta medicação, embora não constasse na tabela institucional, foi prescrita e administrada por hipodermóclise, ocasionando dor no local na inserção do cateter e a necessidade de nova punção. 


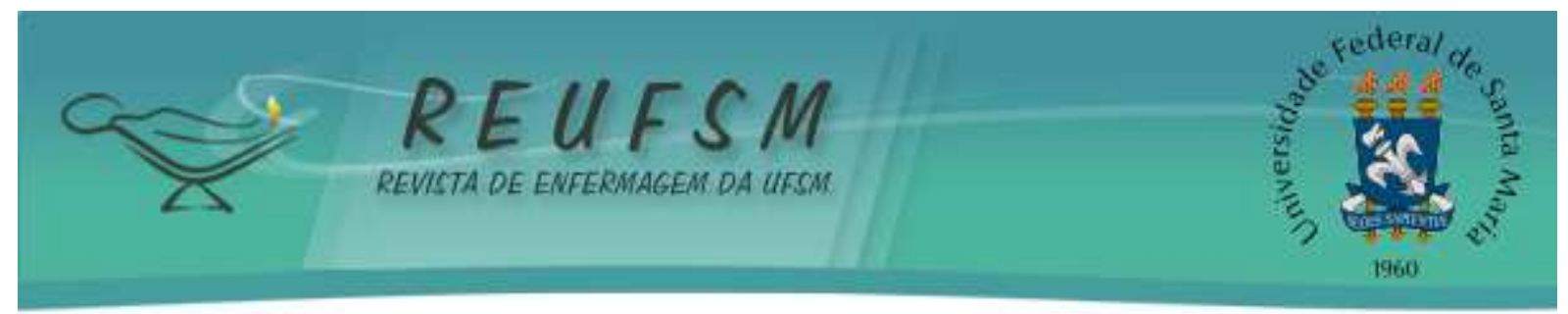

Os pacientes deste estudo utilizaram mais de uma medicação prescrita no mesmo sítio de punção, conforme volume e compatibilidade recomendados para os fármacos. Pesquisas têm sinalizado, ${ }^{3,7,14,16,21,23-25}$ preferencialmente, a escolha de um sítio subcutâneo para cada tipo de medicação e, na vigência de sinais de desconforto, interromper a infusão e realizar nova punção em outro local. O tempo médio de permanência do cateter, neste estudo, foi de 7,25 dias. $\mathrm{Na}$ literatura e em estudos de caso há recomendação de que, no ambiente hospitalar, seja preconizado o tempo de três a cinco dias, podendo permanecer por um período superior a sete dias se não houver sinais de complicações local - eritema, inchaço, dor e extravasamento do fármaco. Não há, no entanto, evidência clínica recente ou estudos brasileiros que indiquem a frequência de troca do cateter e alternância do sítio de punção. ${ }^{1,4,6,8,11,24-25}$

Em relação aos principais motivos de retirada do cateter foram decorrentes da morte do paciente $(59 \%)$, seguido por complicações $(17,2 \%)$, observando-se que as complicações apresentadas foram locais e não sistêmicas, não havendo nenhuma devido a reação adversa severa relacionada aos medicamentos administrados.

Esses resultados apontam para a segurança da via subcutânea no controle dos sinais e sintomas dos pacientes e reforçam a aplicabilidade da hipodermóclise como via parenteral preferencial nas configurações de cuidados paliativos e de fim de vida.

\section{CONCLUSÃO}

Os resultados contribuem para mudança na prática no cenário estudado e trazem embasamento para aumentar o tempo de permanência do dispositivo, para um período de sete dias. Quanto à utilização do fármaco dimenidrinato, tal fato remete à importância de ações educativas permanentes para qualificar e atualizar a equipe multiprofissional, além do incentivo de consulta sistemática das rotinas e protocolos institucionais, os quais visam as melhores práticas e a segurança do paciente.

A hipodermóclise proporcionou aos pacientes com câncer em cuidados paliativos uma terapêutica medicamentosa eficaz, segura e menos invasiva, apresentando-se como uma opção de fácil uso, boa tolerabilidade e baixo risco de complicação para infusões parenterais. Ainda, este estudo contribui para a implementação desta técnica em outros ambientes assistenciais, de forma a beneficiar pacientes elegíveis no uso da terapêutica por hipodermóclise. 


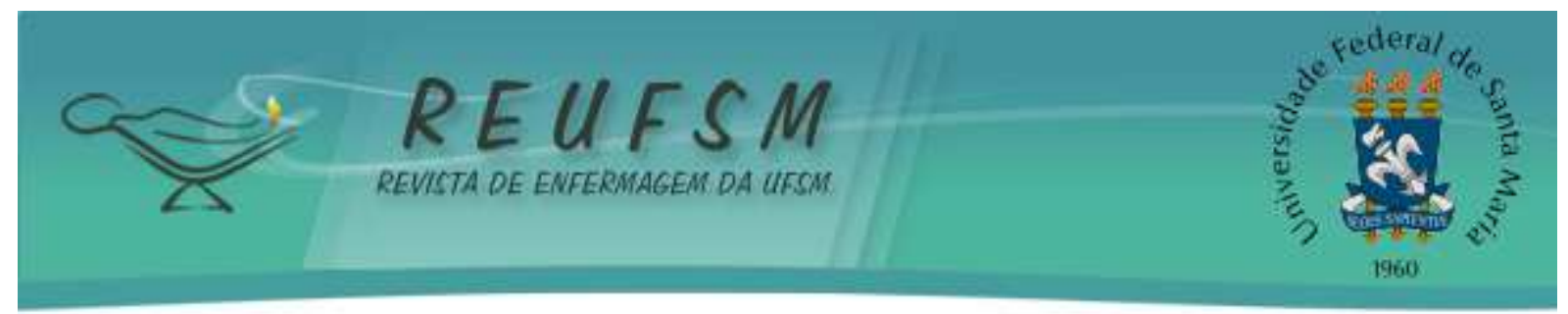

Uma possível limitação deste estudo está relacionada com o viés dos pacientes que tiveram a retirada do dispositivo por motivo de óbito e não por complicações relacionadas ao procedimento, o que pode ter interferido no resultado do tempo de permanência do dispositivo. Sugere-se a realização de estudos clínicos em pacientes com doenças crônicas avançadas para identificar o perfil de segurança dos mesmos em relação à terapêutica em ambiente hospitalar e na continuidade dos cuidados em casa.

\section{REFERÊNCIAS}

1. Cogo ALP, Pontalti G, Echer IC, Santos L, Torriani MS. Vias de administração de medicamentos. In: Torriani MS, Santos L, Echer IC, Barros E, organizadores. Medicamentos de A a Z 2016/2018: Enfermagem 2016. Porto Alegre: Artmed; 2016. p. 40-55.

2. Azevedo EF, Barbosa LA, Cassiani SHB. Administração de antibióticos por via subcutânea: uma revisão integrativa da literatura. Acta Paul Enferm [Internet]. 2012 [acesso 2017 ago 10];25(5):817-22. Disponível em: http://www.scielo.br/pdf/ape/v25n5/26.pdf.

3. Sociedad Española de Cuidados Paliativos (SECPAL). Uso de la vía subcutánea en cuidados paliativos. Espanha; 2013 [acesso 2015 set 23]. 56 p. Disponível em: http://www.secpal.com//Documentos/Blog/monografia_secpal_04.pdf.

4. Braz CL, Pereira RCC, Costa JM. Administração de medicamentos por hipodermóclise: uma revisão da literatura. Rev Bras Farm Hosp Serv Saúde de São Paulo. 2015 [acesso 2017 ago 10];6(1):6-12 [acesso em 2017 ago 10]. Disponivel em: http://www.sbrafh.org.br/rbfhss/public/artigos/2015060102000656BR.pdf.

5. Arthur AO. Innovations in subcutaneous infusions. J Infus Nurs [Internet]. 2015 [acesso 2017 ago 10];38(3):179-87. Disponível em: https://www.ncbi.nlm.nih.gov/pubmed/25871865.

6. Noriega OD, Blasco SA. Subcutaneous fluid and drug delivery: safe, efficient and inexpensive. Rev Clinical Gerontology. 2015 [acesso 2017 ago 10];25(2):117-46. Disponível em: https://doi.org/10.1017/S095925981500012X.

7. Ministério da Saúde. Instituto Nacional do Cancer (INCA). Terapia subcutânea no câncer avançado [Internet]. Rio de Janeiro: INCA; 2009 [acesso 2017 ago 10]. 32p. Disponível em: http://bvsms.saude.gov.br/bvs/publicacoes/inca/Terapia_subcutanea.pdf. (Série Cuidados Paliativos).

8. Pontalti G, Riboldi CO, Gioda RS, Echer IC, Franzoi MA, Wegner W. Benefícios da Hipodermóclise na clínica paliativa de pacientes com câncer: relato de caso. Rev Bras Cancerologia. 2016 [acesso 2017 ago 10];62(3):247-52. Disponível em: http://www1.inca.gov.br/rbc/n_62/v03/pdf/07-relato-de-caso-beneficios-da-hipodermoclisena-clinica-paliativa-de-pacientes-com-cancer.pdf.

9. Pastrana T, Lima L, Centeno-Cortes C, Wenk R, Eisenchlas J, Monti C, Rocafort J. Atlas de cuidados paliativos en latinoamérica. 2012 [acesso 2017 ago 10]. Disponível em: http://dadun.unav.edu/bitstream/10171/34465/1/Atlas\%20de\%20Cuidados\%20Paliativos\%20 de\%20Latinoamerica.pdf. 


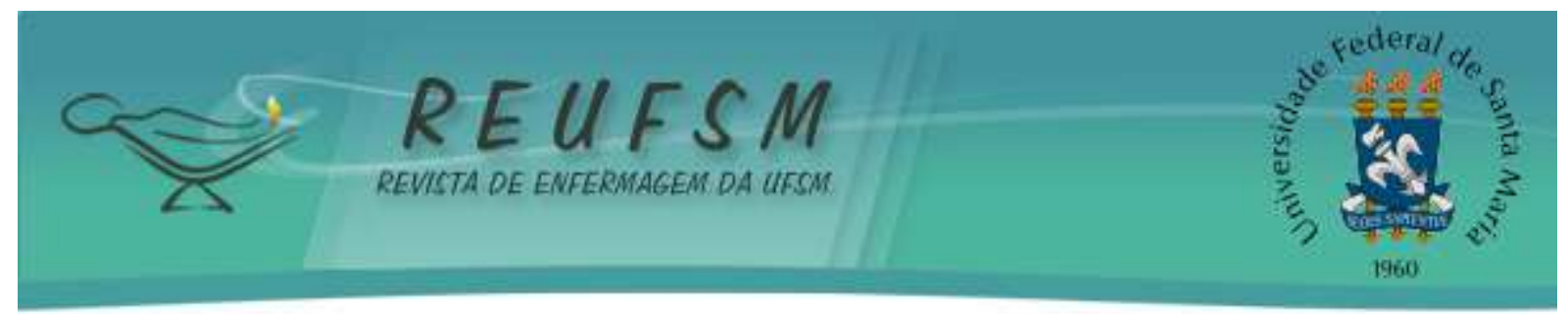

10. Garcia JBS, Rodrigues RF, Lima SF. Structuring a palliative care service in Brazil: experience report. Rev Bras Anestesiol [Internet]. 2014 [acesso 2017 ago 10];64(4):286-291. https://doi.org/10.1016/j.bjan.2013.06.007.

11. Pontalti G, Rodrigues ESA, Firmino F, Fabris M, Stein MR, Longaray VK. Via subcutânea: segunda opção em cuidados paliativos. Rev HCPA [Internet]. 2012[acesso 2017 ago 10];32(2):199-207. Disponível em: http://www.lume.ufrgs.br/bitstream/handle/10183/157903/000871761.pdf?sequence=1.

12. Vidal FKG, Oselame GB, Neves EB, Oliveira ELIA. Hipodermóclise: revisão sistemática da literatura. Rev Atenção Saúde. 2015 [acesso 2017 ago 10];13(45):61-9. Disponivel em: http://seer.uscs.edu.br/index.php/revista_ciencias_saude/article/view/2953/1784

13. Araújo D, Linch GFC. Cuidados paliativos oncológicos: tendências da produção científica. Rev Enferm UFSM [Internet]. 2011 maio/ago;1(2):238-45. Disponível em: https://periodicos.ufsm.br/reufsm/article/view/2482/1636.

14. Azevedo DLO, organizador. O uso de via subcutânea em geriatria e cuidados paliativos: um guia da SBGG e da ANCP para profissionais. Rio de Janeiro: SBGG; 2016 [acesso em 2016 jul 14]. Disponível em: http://sbgg.org.br/wp-content/uploads/2016/06/uso-da-viasubcutanea-geriatria-cuidados-paliativos.pdf.

15. Guidelines for the use of subcutaneous medications in palliative care for adults NHS greater Glasgow, acute services division palliative care practice evelopment [Internet]. 2011 [acesso em 2015 dez 11]. Disponível em: http://www.nhslanarkshire.org.uk/Services/PalliativeCare.

16. Ministério da Saúde. Conselho Nacional de Saúde. Resolução CNS nº466, de 12 de dezembro de 2012. Define as diretrizes e normas reguladoras para as pesquisas envolvendo os seres humanos. Brasília: Diário Oficial da União; 2012 dez 12 [acesso em 2015 set 28]. Disponível em: http://conselho.saude.gov.br/resolucoes/2012/Reso466.pdf.

17. Brasil. Ministério da Saúde. Instituto Nacional de Câncer (INCA). Rio de Janeiro: Ministério da Saúde; 2014 [acesso 2015 nov 19]. Disponível em: http://www.inca.gov.br/rbc/n_60/v01/pdf/11resenha-estimativa-2014-incidencia-de-cancer-no-brasil.pdf.

18. Deandrea S, Montanari M, Moja L, Apolone G. Prevalence of undertreatment in cancer pain: a review of published literature. J. Clin Oncol. 2015;19(12):1985-91. Disponível em: https://academic.oup.com/annonc/article/19/12/1985/167500.

19. Liang S, Tung H, Wu F, Tsay SL, Wang TJ, Chen KP, et al. Concerns about pain and prescribed opioids in Taiwanese oncology outpatients. Pain Manag Nurs. 2013;14(4):336-42. Disponível em: http://www.sciencedirect.com/science/article/pii/S152490421100141X.

20. Matoses CC, Rodríguez FJL, Sanz GT, Murcia ACL, Morante MH, Navarro AR. Administración de medicamentos por vía subcutánea en cuidados paliativos. Farmacia Hospitalaria [Internet]. 2015 [acesso 2017 ago 10];39(2):71-9. Disponivel em: http://scielo.isciii.es/pdf/fh/v39n2/02original01.pdf.

21. Fonzo CF, Vukasovic C, Wasilewski RAF, Bonnabry P. Subcutaneous administration of drugs in the elderly: survey of practice and systematic literature review. Palliative Medicine. 2005 [acesso 2016 jan 22];19(3):208-19. Disponível em: http://journals.sagepub.com/toc/pmja/19/3. 


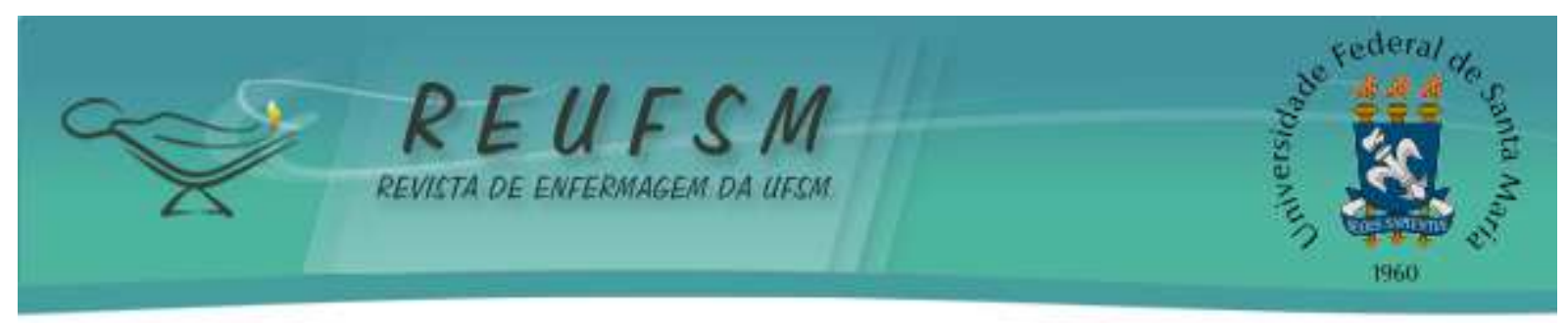

22. Nunes PMSA, Souza RCSS. Efeitos adversos da hipodermóclise em pacientes adultos: revisão integrativa. Rev Min Enferm [Internet]. 2016 [acesso em 2015 set 23];20. Disponível em: http://www.reme.org.br/exportar-pdf/1084/e951.pdf.

23. Academia Nacional de Cuidados Paliativos (ANCP). Manual de cuidados paliativos ANCP [Internet]. São Paulo. 2012 [acesso em 2015 set 23]. 592 p. Disponível em: http://www.paliativo.org.br/biblioteca virtual.

24. Tejedor DF, Alvarez AIP. Estudio de la utilización de la vía subcutánea en los pacientes ingresados en una unidad de cuidados paliativos. Rev Enferm CyL. 2010 [acesso em 2015 set 23];1(2):2-8.

Disponível

em: http://www.revistaenfermeriacyl.com/index.php/revistaenfermeriacyl/article/viewFile/35/26.

25. Bruno VG. Hipodermóclise: revisão de literatura para auxiliar a prática clínica. São Paulo: Einstein; 2013 [acesso em 2017 set 20];2(7). Disponível em: http://www.scielo.br/pdf/eins/2015nahead/pt_1679-4508-eins-1679-45082015RW2572.pdf.

Data de submissão: 10/08/2017

Data de aceite: 07/05/2018

Autor correspondente: Gislene Pontalti

Email: gislene818@gmail.com

Endereço: Veríssimo Rosa, 321/201. Bairro Partenon, Porto Alegre/RS

CEP: 90610-280 